\title{
Estudo químico da fração diclorometânica do extrato de Ocimum gratissimum $\mathrm{L}$.
}

\section{Phytochemical investigation of dichloromethanic fraction on Ocimum gratissimum L. extract}

\author{
Michele Rosset ${ }^{1}$; Vitor de Moraes Zamarion ${ }^{1}$; Milton Faccione ${ }^{2}$; Terezinha de Jesus \\ Faria ${ }^{2}$; Jurandir Pereira Pinto ${ }^{3}$; Aneli de Melo Barbosa ${ }^{4}$; José Roberto Pinto de Souza ${ }^{5 *}$
}

\section{Resumo}

\begin{abstract}
Ensaios anteriores de atividade antifúngica do extrato etanólico de Ocimum gratissimum, utilizando a técnica de difusão em meio sólido, evidenciaram a inibição do crescimento de Penicillium chrysogenum, Rhizoctonia sp, Aspergillus niger e de duas espécies de Alternaria sp isoladas de tomate e de cenoura. O objetivo deste trabalho foi identificar os possíveis constituintes químicos responsáveis pela atividade antifúngica da planta, por meio de estudo químico do extrato hexano-diclorometano-etanol (1:3:1) das partes aéreas de $O$. gratissimum. O extrato foi cromatografado em coluna de sílica gel, eluída com hexano, diclorometano e etanol. A purificação em coluna cromatográfica de sílica gel das frações eluídas com diclorometano $(5,9 \mathrm{~g})$ resultou na obtenção de quatro constituintes: uma mistura de hidrocarbonetos saturados de cadeia longa $\left(\mathrm{C}_{31}, \mathrm{C}_{33}, \mathrm{C}_{34} \mathrm{e}_{35}\right)$, eugenol, óxido de cariofileno e uma mistura de estigmasterolsitosterol. Os constituintes foram identificados através das análises por cromatografia gasosa acoplada à espectrometria de massas e das espectrometrias no infravermelho, de ressonância magnética nuclear de ${ }^{1} \mathrm{He}$ de ${ }^{13} \mathrm{C}$ e comparação com dados espectrais da literatura. Entre os constituintes isolados, concluiuse que o eugenol é um dos prováveis responsáveis pela atividade antifúngica de $O$. gratissimum, pois registros da literatura comprovaram a atividade deste constituinte contra várias espécies de fungos.

Palavras-chave: Ocimum gratissimum, atividade antifúngica, eugenol, fungos fitopatogênicos
\end{abstract}

\begin{abstract}
Previous studies showed that ethanolic extract of Ocimum gratissimum inhibited growth of Penicillium chrysogenum, Rhizoctonia sp, Aspergillus niger and Alternaria sp, isolated from tomato and carrot. The aim of this study was to identify the chemical constituents of Ocimum gratissim. Aerial parts of the plant were treated with hexane-dichloromethane-ethanol mixture (1:3:1) at room temperature. The obtained extract was chromatographed on silica gel column eluted with hexane, dichloromethane and ethanol. Fractions eluted with dichloromethane were rechromatographed on a column of silica gel to yield alyphatic hydrocarbon mixture $\left(\mathrm{C}_{31}, \mathrm{C}_{33}, \mathrm{C}_{34}, \mathrm{C}_{35}\right)$, eugenol, caryophyllene oxide and a mixture of stigmasterol and sitosterol. The structures of the compounds were deduced by spectral data analysis and by comparison with literature values. Recent studies showed that eugenol is probably one of the responsibles for antifungal activity of $O$. gratissimum.
\end{abstract}

Key words: Ocimum gratissimum, antifungal activity, eugenol, phytopathogenic fungi

1 Aluno de Graduação do Curso de Química, Departamento de Química, UEL, Londrina -PR

2 Professor Dr., Departamento de Química, CCE, UEL, Londrina -PR

3 Técnico M.Sc, Departamento de Química, CCE, UEL, Londrina -PR

4 Professora Dr., Departamento de Bioquímica, CCE, UEL, Londrina -PR

5 Professor Dr., Departamento de Agronomia, CCA, UEL, Londrina -PR

* Autor para correspondência.

Recebido para publicação 16/12/04 Aprovado em 08/07/05 


\section{Introdução}

Os fitopatógenos representam um dos fatores limitantes da produção de alimento, tornando necessária a utilização de agrotóxicos para garantir a produtividade e a qualidade dos produtos. Entretanto, devido à toxicidade e alto poder residual, os defensivos agrícolas sintéticos têm provocado contaminação do ambiente e danos à saúde humana (SANTOS, 1999). Estes fatos, aliados ao desenvolvimento da resistência de microrganismos aos agentes antimicrobianos, estimularam a pesquisa de novas substâncias capazes de impedir o crescimento de fungos e bactérias.

As espécies vegetais representam um campo a ser mais explorado na busca de novas substâncias antifúngicas, visto que a maioria dos trabalhos registrados na literatura sobre antifúngicos naturais tratam de metabólitos obtidos de microrganismos. Várias classes de substâncias do metabolismo secundário de plantas apresentam ação antimicrobiana . Como exemplo pode-se citar a classe dos isoflavonóides, que atuam como fitoalexinas, representando, portanto, um mecanismo natural de resistência das plantas ao ataque de fungos e bactérias (SMITH; BANKS, 1986). Por serem naturais e possuírem atividade antimicrobiana altamente específica, os isoflavonóides podem ser uma alternativa aos antifúngicos convencionais na estocagem de grãos (WEIDENBÖRNER et al., 1990) e no controle de fungos nas áreas de produção agrícola. Esta é uma alternativa importante, principalmente nos países em desenvolvimento, onde a utilização de agrotóxicos eleva os custos de produção.

Ocimum gratissimum, pertencente à família Labiatae, possui ampla distribuição nas regiões tropicais de clima quente (PESSOA et al., 2002). A planta é utilizada popularmente no Brasil e na África para diversos fins terapêuticos como tratamento de dores de cabeça, diarréia, pneumonia, problemas estomacais, tosse, febre e conjuntivite (ONAJOBI, 1986; CORRÊA, 1926). Estudos registrados na literatura comprovaram várias propriedades biológicas do óleo essencial de O.gratissimum, entre elas as atividades antifúngica (DUBEY et al., 2000; NAKAMURA et al., 2004), antibacteriana (JANSSEN et al., 1989; NAKAMURA et al., 1999; CIMANGA et al., 2002; NGASSOUM et al., 2003) e inseticida (KÉITA et al., 2001). Extratos da planta apresentaram atividade antidiarréica (ONAJOBI, 1986; OFFIAH; CHIKWENDU, 1999), hipoglicêmica em ratos (AGUIYI et al., 2000) e contra os vírus HIV-1 e HIV-2 (AYISI; NYADEDZOR, 2003). Ensaios biológicos realizados no Departamento de Bioquímica da UEL indicaram atividade antifúngica do extrato etanólico de $O$. gratissimum contra os fungos Penicillium chrysogenum, Rhizoctonia sp, Aspergillus niger, duas espécies de Alternaria sp isoladas de tomate e de cenoura. Apesar dos vários registros sobre as atividades biológicas da espécie, existem poucas informações sobre os constituintes químicos de extratos desta espécie e sobre a relação destas substâncias com as propriedades biológicas da planta.

Com base nos fatos apresentados, foi iniciado o estudo químico de $O$. gratissimum, visando identificar os constituintes químicos responsáveis pela atividade antifúngica do extrato bruto da planta.

\section{Material e Métodos}

Para análise e isolamento das substâncias, o material vegetal foi desidratado. $\mathrm{O}$ extrato, obtido pela extração com mistura de hexano/diclorometano/ etanol 1:3:1 das partes aéreas de O. gratissimum desitratadas, foi fracionado em coluna de sílica gel, eluída com hexano, diclorometano e etanol. O isolamento e a identificação dos constituintes químicos foram realizados no material resultante das frações eluídas com diclorometano (fração diclorometânica), de acordo com as seguintes fases:

Material vegetal: Ocimum gratissimum foi cultivada no Horto de Plantas Medicinais do Departamento de Agronomia do Centro de Ciências Agrárias (CCA) da Universidade Estadual de 
Londrina e está registrada no Herbário da mesma Universidade sob número 36554. Após a coleta, as partes aéreas da planta foram submetidas a processo de secagem em estufa a $40^{\circ} \mathrm{C}$ e, em seguida, foram trituradas, fornecendo $429 \mathrm{~g}$ de material seco.

Extração e fracionamento do extrato: $\mathrm{O}$ material vegetal $(429 \mathrm{~g})$ foi submetido à extração com hexano/diclorometano/etanol na proporção de 1:3:1. O solvente de extração foi escolhido com base em um planejamento experimental para escolha do melhor solvente extrator. Eliminação do solvente por destilação à pressão reduzida resultou na obtenção de $25 \mathrm{~g}$ de extrato, o qual foi cromatografado em coluna filtrante de sílica gel, eluída com hexano, diclorometano e etanol. O grupo de frações eluído com diclorometano forneceu $5,90 \mathrm{~g}$ de material.

Isolamento dos constituintes químicos da fração diclorometânica: O material resultante do grupo de frações eluídas com diclorometano $(5,90 \mathrm{~g})$ foi purificado em coluna de sílica gel, utilizando como eluentes hexano, diclorometano, acetato de etila e etanol em misturas com gradientes crescentes de polaridade. Foram coletadas 118 frações de $250 \mathrm{~mL}$, as quais foram reunidas em 25 grupos por comparação em placas cromatográficas de sílica gel. O grupo frações 1-7, eluído com hexano, forneceu um sólido branco $(67,6 \mathrm{mg})$, ponto de fusão $88^{\circ} \mathrm{C}$ $90^{\circ} \mathrm{C}$, identificado como uma mistura de hidrocarbonetos saturados. O grupo de frações 2126 , eluído com hexano/diclorometano $15 \%$, foi purificado em coluna de sílica gel e em placas preparativas, também de sílica gel.

Cromatogragia gasosa acoplada ao espectrômetro de massas: A cromatografia gasosa acoplada ao espectrômetro de massas foi realizada em equipamento Shimadzu, modelo GC- 17A, com detetor seletivo de massa, modelo QP 5000 (Shimadzu). A coluna cromatográfica utilizada foi do tipo capilar de sílica fundida com fase estacionária DB-1 (polimetisiloxano), de 30m de comprimento e $0,25 \mathrm{~mm}$ de diâmetro interno, utilizando hélio como gás de arraste. As temperaturas foram de $300^{\circ} \mathrm{C}$ no injetor e $250^{\circ} \mathrm{C}$ no detetor. A temperatura do forno foi programada de 60 a $250^{\circ} \mathrm{C}$, com acréscimo de $10^{\circ} \mathrm{C}$ a cada minuto e de $250^{\circ} \mathrm{C}$ a $320^{\circ}$ com acréscimo de $20^{\circ} \mathrm{C}$ a cada minuto.

Identificação do número de átomos de carbono dos constituintes da mistura de hidrocarbonetos: A identificação do número de átomos de carbono dos hidrocarbonetos da mistura, foi feita através de cromatografia gasosa da mistura e de padrões de

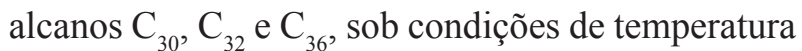
programada na faixa de $100 \mathrm{a} 340^{\circ} \mathrm{C}$. As temperaturas foram de $250^{\circ} \mathrm{C}$ no injetor e de $230^{\circ} \mathrm{C}$ no detetor. Os dados fornecidos pelo cromatograma dos padrões foram utilizados na construção do gráfico Temperatura de retenção x Número de átomos de carbono. O número de átomos de carbono de cada alcano da mistura foi determinado através da temperatura de retenção e dos parâmetros A e B obtidos do gráfico.

\section{Resultados e Discussões}

O resultado da purificação do material resultante da fração diclorometânica por métodos cromatográficos usuais, forneceu duas substâncias de aspecto oleoso, identificadas como eugenol (Figura 1A) e óxido de cariofileno (Figura 1B), uma mistura de hidrocarbonetos alifáticos e uma mistura dos esteróides estigmasterol e sitosterol. $\mathrm{O}$ eugenol foi identificado através das análises por cromatografia gasosa, acoplada à espectrometria de massas e das espectrometrias no infravermelho e de ressonância magnética nuclear de ${ }^{1} \mathrm{H}$ e de ${ }^{13} \mathrm{C}$ e pela comparação dos dados espectrais com os dados registrados na literatura para o eugenol (SAITO et al., 2004). Os demais constituintes foram identificados através da análise por cromatografia gasosa, acoplada à espectrometria de massas. A determinação do número de átomos de carbono dos constituintes da mistura de hidrocarbonetos indicou tratar-se de uma mistura de hidrocarbonetos alifáticos saturados $\mathrm{C}_{31}$, $\mathrm{C}_{33}, \mathrm{C}_{34}$ e $\mathrm{C}_{35}$. 


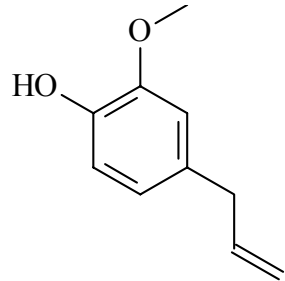

(A)

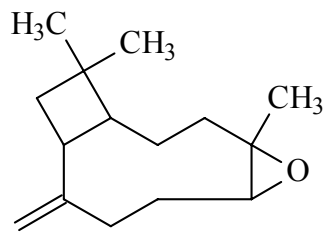

(B)
Figura 1. Representação das estruturas de algumas substâncias encontradas no extrato da parte aérea de $O$. gratissimum: eugenol (A) e óxido de cariofileno (B).

O total de eugenol obtido foi $28,3 \mathrm{mg}$ e o total de óxido de cariofileno foi $1,3 \mathrm{mg}$. O grupo de frações 42-46, eluído com hexano/diclorometano $30 \%$, ao ser purificado em coluna cromatográfica de sílica gel, forneceu um sólido branco $(16,8 \mathrm{mg})$, cuja análise por cromatografia gasosa acoplada à espectrometria de massas indicou tratar-se de uma mistura dos esteróides estigmasterol e sitosterol.

Entre os constituintes isolados, concluiu-se que o eugenol deve ser um dos prováveis responsáveis pela atividade antifúngica do extrato de $O$. gratissimum, pois registros da literatura comprovaram a atividade deste constituinte contra várias espécies de fungos (DUBEY et al., 2000; NAKAMURA et al., 2004).

Outros estudos estão sendo realizados para se identificar outros possíveis substâncias antifúngicas das demais frações (hexânica e etanólica) obtidas do fracionamento do extrato bruto de $O$. gratissimum.

\section{Agradecimentos}

Os autores agradecem o apoio do Conselho Nacional de Desenvolvimento Científico e Tecnológico (CNPq), da Fundação Araucária e da Universidade Rural do Rio de Janeiro.

\section{Referências}

AGUIYI, J. C.; OBI, C. I.; GANG, S. S.; IGWEH, A. C. Hypoglycaemic activity of Ocimum gratissimum in rats. Fitoterapia, Milano, v.71, n.4, p.444-446, 2000.
AYISI, N. K.; NYADEDZOR, C. Comparative in vitro effects of AZT and extracts of Ocimum gratissimum, Ficus polita, Clausena anisata, Alchornea cordifolia, and Elaeophorbia drupifera against HIV-1 and HIV-2 infections. Antiviral Research, Amsterdam, v.58, n.1, p.2533, mar. 2003.

CIMANGA, K.; KAMBU, L.; TONA, L.; APERS, S.; BRUYNE, T.; HERMANS, N.; TOTTÉ, J.; PIETERS, L.; VLIETINCK, A. J. Correlation between chemical composition and antibacterial activity of essential oils of some aromatic medicinal plants growing in the Democratic Republic of Congo. Journal of Ethnopharmacology, Lausanne, v. 79, n.2, p.213-220, Feb. 2002.

CORRÊA, M. P. Dicionário das plantas úteis do Brasil e das exóticas cultivadas. Rio de Janeiro: Imprensa Nacional, 1926. v.1.

DUBEY, K. N.; TIWARI, T. N.; MANDIN, D.; ANDRIAMBOAVONJY, H.; CHAUMONT, J. P. Antifungal properties of Ocimum gratissimum essential oil (ethyl cinnamate chemotype). Fitoterapia, Milano, v.71, p.567569,2000 .

JANSSEN, A. M.; SCHEFFER, J. J. C.; NTEZURBANZA, L.; SVENDSEN, A. B. Antimicrobial activities of some Ocimum species grown in Rwanda. Journal of Ethnopharmacology, Lausanne, v.26, p.57-63, 1989.

KÉITA, S. M.; VICENT, C.; SCHMIT J. P.; ARNASON, J. T.; BÉLANGER, A. Efficacy of essential oil of Ocimum gratissimum L. and $O$. gratissimum L. applied as an insecticidal fumigant and powder to control Callosobruchus maculalus (Fab.) [Coleoptera: Bruchidae]. Journal of Stored Products Research, Oxford, v.37, p.339349, 2001.

NAKAMURA, C. V.; UEDA-NAKAMURA, T.; BANDO, E.; MELO, N. A. F. N.; CORTEZ, D. A. G.; DIAS FILHO, B. P. Antibacterial Activity of Ocimum gratissimum L. essential oil. Memórias do Instituto Oswaldo Cruz, Rio de Janeiro, v.94. n.5, p.675-678, Sect./Out. 1999.

NAKAMURA, C. V.; ISHIDA, K.; FACCIN, L. C.; DIAS FILHO, B. P; CORTEZ, D. A. G.; ROZENTAL, S.; SOUZA, W.; UEDA-NAKAMURA, T. In viro activity of essential oil from Ocimum gratissimum L. against four Candida species. Research in Microbiology, Paris, v. 155, p.579586,2004

NGASSOUM, M. B.; ESSIA-NGANG, J. J.; TATSADJIEU, L. N.; JIROVETZ, L.; BUCHBAUER, G; ADJOUDJI, O. Antimicrobial study of essential oils of Ocimum gratissimum leaves and Zanthoxylum xanthoxyloides fruits from Cameroon. Fitoterapia, Milano, v.74, n.3, p.284287, 2003. 
OFFIAH, V. N.; CHIKWENDU, U. A. Antidiarrhoeal effects of Ocimum gratissimum leaf extract in experimental animals. Journal of Ethnopharmacology, Lausanne, v.68, n.1/3, p.327-330, 1999.

ONAJOBI, F. D. Smooth muscle contracting lipid-soluble principles in chromatographic fractions of Ocimum gratissimum. Journal of Ethnopharmacology, Lausanne, v.18, n.1, p.3-11,1986.

PESSOA, L. M.; MORAIS, S. M.; BEVILAQUA, C. M. L.; LUCIANO, J. H. S. Anthelmintic activity of essential oil of Ocimum gratissimum Linn. and eugenol against Haemonchus contortus. Veterinary Parasitology, Amsterdam, v.109, n.1/2, p.59-63, 2002.
SAITO, T.; HAYAMIZU, K.; YANAGISAWA M.; YAMAMOTO, O.; WASADA, N.; KINUGASA, S.; TANABE, K.; TAMURA, T. Integrated spectral data base system for organic compounds. Disponível em: <http:// www.aist.go.jp/RIODB/SDBS/.> Acesso em: 30 set.2004.

SANTOS, S. R. Dos espirros à morte: agrotóxicos provocam mortes e danos irreversíveis à saúde humana. Revista CREA/PR, Curitiba, v.1, n.4, p.22-23, 1999.

SMITH, D. A.; BANKS, S. W. Biosynthesis, elicitation and biological activity of isoflavonoids phytoalexins. Phytochemistry, New York, v.25, n.5, p.979-995,1986.

WEIDENBÖRNER, M.; HINDORF, H.; JHA, H. C.; TSOTSONOS, P. Antifungal activity of flavonoids against storage fungi of the Genus Aspergillus. Phytochemistry, New York, v.29, n.4, p.1103-1105, 1990. 